\title{
Time Scales of a Chaotic Semiconductor Laser With Optical Feedback Under the Lens of a Permutation Information Analysis
}

\author{
Miguel C. Soriano, Luciano Zunino, Osvaldo A. Rosso, Ingo Fischer, and Claudio R. Mirasso
}

\begin{abstract}
We analyze the intrinsic time scales of the chaotic dynamics of a semiconductor laser subject to optical feedback by estimating quantifiers derived from a permutation information approach. Based on numerically and experimentally obtained times series, we find that permutation entropy and permutation statistical complexity allow to extract important characteristics of the dynamics of the system. We provide evidence that permutation statistical complexity is complementary to permutation entropy, giving valuable insights into the role of the different time scales involved in the chaotic regime of the semiconductor laser dynamics subject to delay optical feedback. The results obtained confirm that this novel approach is a conceptually simple and computationally efficient method to identify the characteristic time scales of this relevant physical system.
\end{abstract}

Index Terms-Semiconductor lasers, optical feedback, chaos, time scale identification, permutation entropy, permutation statistical complexity.

\section{INTRODUCTION}

The identification of essential physical time scales from complex laser dynamics is a nontrivial task, which is however important for their general characterization and application. In particular, systems with time delays can generate chaotic dynamics with high complexity, i.e. they posses a large number of dynamical degrees of freedom [1]. This is one of the properties which makes delay systems very attractive for applications. Particularly, optical chaos encryption is based on the unpredictability of the chaotic carrier [2] besides its synchronizability [3]. Chaotic radar [4] and lidar [5], rainbow refractometry [6], and ultrahigh-speed physical random number generation [7], [8] are other relevant applications of optical chaos based on delay-phenomena. Semiconductor lasers with optical feedback have been shown to be particularly suitable for these applications due to their large dynamical bandwidth [9]-[13]. This bandwidth amounts to typically several $\mathrm{GHz}$, related to

M. C. Soriano, L. Zunino, I. Fischer and C. R. Mirasso are with the Instituto de Física Interdisciplinar y Sistemas Complejos (IFISC) CSIC-UIB, Campus Universitat de les Illes Balears, E-07122 Palma de Mallorca, Spain (e-mail: miguel@ifisc.uib-csic.es; luciano@ifisc.uib-csic.es; ingo@ifisc.uibcsic.es; claudio@ifisc.uib-csic.es).

L. Zunino is also with the Centro de Investigaciones Ópticas (CONICET La Plata - CIC), C.C. 3, 1897 Gonnet, Argentina and Departamento de Ciencias Básicas, Facultad de Ingeniería, Universidad Nacional de La Plata (UNLP), 1900 La Plata, Argentina (e-mail: lucianoz@ciop.unlp.edu.ar).

O. A. Rosso is with the Instituto de Ciências Exatas (Fisica), Universidade Federal de Minas Gerais, Av. Antônio Carlos, 6627 - Campus Pampulha, 31270-901 Belo Horizonte - MG, Brazil and Chaos \& Biology Group, Instituto de Cálculo, Facultad de Ciencias Exactas y Naturales, Universidad de Buenos Aires, Pabellón II, Ciudad Universitaria. 1428 Ciudad de Buenos Aires, Argentina (e-mail: oarosso@fibertel.com.ar). the relaxation oscillation period of the semiconductor laser, but possibly also faster time scales, as we will discuss in this paper. The resolution of chaotic lidar and the transmission rates of chaos communications are limited by this characteristic fast time scale of the semiconductor laser [14]. The feedback time delay is another intrinsic time scale determining the dynamics of semiconductor lasers with feedback. The time delay is important to generate suitable carriers for chaos communication, but also, because the dynamics of certain chaotic delayed systems can be identified and modeled once their time delay is known [15]-[17]. Consequently, the identification of the time delay could compromise the security and confidentiality of chaotic communication systems [18]-[20]. Rontani et al. [21], [22] have recently shown that difficult time delay identification scenarios strongly depend on the time scales of the system, i.e. the separation between the relaxation oscillation period and feedback time delay plays a starring role in the retrieval of the time delay.

For all these aspects, a detailed study of the time scales present in the chaotic dynamic of a semiconductor laser subject to optical feedback is very important. This critical issue is addressed in this work by estimating permutation entropy, $\mathcal{H}_{S}$, and permutation statistical complexity, $\mathcal{C}_{J S}$, of both, numerical and experimental time series of the laser output power as functions of the embedding delay $\tau$ of a particular symbolic reconstruction. It is worth mentioning that this novel approach, derived from information theory, provides useful evidence about time delay phenomena present in noisy time series [23]. More specifically, in this work it is found that both quantifiers, $\mathcal{H}_{S}$ and $\mathcal{C}_{J S}$, develope clear extrema when the embedding delay $\tau$ matches the characteristic time delay $\tau_{S}$ of the system. In the present work we verify from numerical and experimental time series that these quantifiers are able to identify the feedback time delay and relaxation oscillation period in the dynamics of the semiconductor laser subject to optical feedback operating in a chaotic regime. Additionally, the approach detects an even faster time scale that we relate to fast chaotic dynamical processes. Several implications, in particular temporal detection requirements, are being discussed. We note that, according to our knowledge, we present the first application of this methodology to experimental time series.

The paper is organized as follows. In Section II we describe the two information theory quantifiers estimated in our analysis: permutation entropy, $\mathcal{H}_{S}$, and permutation statistical complexity, $\mathcal{C}_{J S}$. In Sections III and IV, numerical and experimental results, respectively, are presented and discussed. 
Finally, some concluding remarks are given in Section V.

\section{INFORMATION THEORY QUANTIFIERS}

Deterministic chaotic time series produced by nonlinear time delay systems share several properties with those generated by stochastic processes, e.g. a wide-band power spectrum and a long-term unpredictable behavior. They can be hard to distinguish in practical situations and several works aimed at elucidating the deterministic or random nature of a time series [24], [25]. This similarity justifies the use of standard statistical operators to study the properties of chaotic time series. Autocorrelation function (ACF) and delayed mutual information (DMI) are conventional techniques widely used to identify time delays [18], [20]-[22], [26], [27]. However, new alternatives were introduced in recent years in order to perform this task [28]-[35]. We are particularly interested in the application of a permutation information theory methodology to unveil delay phenomena from time series introduced recently [23]. In this approach, quantifiers derived from information theory, more precisely Shannon entropy and statistical complexity, are estimated by using an efficient symbolic technique, the Bandt and Pompe permutation method [36], to determine the probability distribution associated to the time series under study. This way of symbolizing time series, based on a comparison of consecutive points, allows a more accurate empirical reconstruction of the underlying phase space of chaotic time series affected by weak (observational and dynamical) noise [36]. This is the main advantage with respect to standard methods, like ACF and DMI, that take the exact metric into account. Moreover, the ordinal pattern distribution is invariant with respect to nonlinear monotonous transformations. Thus, nonlinear drifts or scalings artificially introduced by a measurement device do not modify the quantifiers estimations. This property is highly desired for the analysis of experimental data. The basic intrinsic structure of complex systems is obtained in a very fast and flexible way. Characteristic time scales present in the system dynamics are detected through the presence of clear extrema of the quantifiers when they are calculated as a function of the embedding delay.

\section{A. Shannon entropy and statistical complexity}

Shannon entropy is widely used as a first natural approach to quantify the information content of a system. Given any arbitrary probability distribution $P=\left\{p_{i}: i=1, \ldots, M\right\}$, the widely known Shannon's logarithmic information measure defined by $S[P]=-\sum_{i=1}^{M} p_{i} \ln p_{i}$ is regarded as the measure of the uncertainty associated to the physical process described by $P$. If $S[P]=0$ our knowledge of the underlying process described by the probability distribution is maximal. In contrast, our knowledge is minimal for a uniform distribution.

However, entropy measures do not quantify the degree of structure or patterns present in a process and measures of statistical or structural complexity are necessary to capture properties related to organization [37]. The opposite extremes of perfect order and maximal randomness (a periodic sequence and a fair coin toss, for example) possess no complex structure.
These systems are defined to have zero statistical complexity. At a given distance from these extremes, a wide range of possible degrees of physical structure exists, that should be quantified by the statistical complexity measure. Lamberti et al. [38] introduced an effective statistical complexity measure (SCM) that is able to detect essential details of the dynamics and differentiate different degrees of periodicity and chaos. This statistical complexity measure is defined, following the intuitive notion advanced by López-Ruiz et al. [39], through the product

$$
\mathcal{C}_{J S}[P]=\mathcal{Q}_{J}\left[P, P_{e}\right] \mathcal{H}_{S}[P]
$$

of the normalized Shannon entropy

$$
\mathcal{H}_{S}[P]=S[P] / S_{\max }
$$

with $S_{\max }=S\left[P_{e}\right]=\ln M,\left(0 \leq \mathcal{H}_{S} \leq 1\right)$ and $P_{e}=\{1 / M, \ldots, 1 / M\}$ the uniform distribution, and the disequilibrium $\mathcal{Q}_{J}$ defined as $\mathcal{Q}_{J}\left[P, P_{e}\right]=\mathcal{Q}_{0} \mathcal{J}\left[P, P_{e}\right]$. $\mathcal{J}\left[P, P_{e}\right]=\left\{S\left[\left(P+P_{e}\right) / 2\right]-S[P] / 2-S\left[P_{e}\right] / 2\right\}$ is the JensenShannon divergence and $\mathcal{Q}_{0}$ a normalization constant, equal to the inverse of the maximum possible value of $\mathcal{J}\left[P, P_{e}\right]$. This maximum value is obtained when one of the components of $P$, say $p_{m}$, is equal to one and the remaining components are equal to zero. The Jensen-Shannon divergence, that quantifies the difference between two (or more) probability distributions, is especially useful to compare the symbol composition between different sequences [40]. We stress the fact that the above SCM is not a trivial function of the entropy because it depends on two different probabilities distributions, the one associated to the system under analysis, $P$, and the uniform distribution $P_{e}$. Furthermore, it has been shown that for a given $\mathcal{H}_{S}$ value, there exists a range of possible SCM values [41]. Thus, it is clear that important additional information related to the correlational structure between the components of the physical system is provided by evaluating the statistical complexity [42], [43].

\section{B. Bandt and Pompe symbolization method}

In order to evaluate the two above-mentioned quantifiers, $\mathcal{H}_{S}$ and $\mathcal{C}_{J S}$, an associated probability distribution should be constructed beforehand. The adequate way of choosing the probability distribution associated to a time series is an open problem. Rarely, a univocal procedure imposes itself. Bandt and Pompe [36] introduced a successful method to evaluate the probability distribution taking into account the time causality of the system dynamics. They took partitions by comparing the order of neighboring values rather than partitioning the amplitude into different levels. That is, given a time series $\left\{x_{t}, t=1, \ldots, N\right\}$, an embedding dimension $D>1$, and an embedding delay time $\tau$, the ordinal pattern of order $D$ generated by

$$
s \mapsto\left(x_{s-(D-1) \tau}, x_{s-(D-2) \tau}, \ldots, x_{s-\tau}, x_{s}\right)
$$

has to be considered. To each time $s$ we assign a $D$ dimensional vector that results from the evaluation of the time series at times $s-(D-1) \tau, \ldots, s-\tau, s$. Clearly, the higher the value of $D$, the more information about the past is 
incorporated into the ensuing vectors. By the ordinal pattern of order $D$ related to the time $s$ we mean the permutation $\pi=\left(r_{0}, r_{1}, \cdots, r_{D-1}\right)$ of $(0,1, \cdots, D-1)$ defined by

$$
x_{s-r_{0} \tau} \geq x_{s-r_{1} \tau} \geq \cdots \geq x_{s-r_{D-2} \tau} \geq x_{s-r_{D-1} \tau} .
$$

In this way the vector defined by Eq. (3) is converted into a unique symbol $\pi$. The procedure can be better illustrated by a simple example; let us assume that we start with the time series $\{3,2,5,1,4,6, \ldots\}$, and we choose the embedding dimension as $D=4$ and the embedding delay as $\tau=1$. In this case the state space is divided into 4 ! partitions and 24 mutually exclusive permutation symbols are considered. The first 4-dimensional vector is $(3,2,5,1)$. According to Eq. (3) this vector corresponds to $\left(x_{s-3}, x_{s-2}, x_{s-1}, x_{s}\right)$. Following Eq. (4) we find that $x_{s-1} \geq x_{s-3} \geq x_{s-2} \geq x_{s}$. Then, the ordinal pattern allowing us to fulfill Eq. (4) will be $(1,3,2,0)$. The second 4-dimensional vector is $(2,5,1,4)$, and $(2,0,3,1)$ will be its associated permutation, and so on. For all the $D$ ! possible permutations $\pi_{i}$ of order $D$, their associated relative frequencies can be naturally computed by the number of times this particular order sequence is found in the time series divided by the total number of sequences. Thus, an ordinal pattern probability distribution $P=\left\{p\left(\pi_{i}\right), i=1, \ldots, D !\right\}$ is obtained from the time series. This probability distribution is derived once we fix the embedding dimension $D$ and the embedding delay time $\tau$. The former parameter plays an important role for the evaluation of the appropriate probability distribution, since $D$ determines the number of accessible states, given by $D$ !. Moreover, it was established that the length $N$ of the time series must satisfy the condition $N \gg D$ ! in order to obtain a reliable statistics [44]. With respect to the selection of the other parameter, Bandt and Pompe specifically considered an embedding delay $\tau=1$ in their cornerstone paper [36]. Nevertheless, it is clear that other values of $\tau$ could provide additional information. It has been recently shown that the embedding delay $\tau$ is strongly related, if it is relevant, with the intrinsic time delay of the system under analysis [23].

In this work the normalized Shannon entropy, $\mathcal{H}_{S}$ (Eq. (2)), and the SCM, $\mathcal{C}_{J S}$ (Eq. (1)), are evaluated using the permutation probability distribution, $P=\left\{p\left(\pi_{i}\right), i=1, \ldots, D !\right\}$. Defined in this way, these quantifiers are usually known as permutation entropy and permutation statistical complexity [45], [46]. These symbolic quantifiers were shown to be particularly useful for different purposes like distinguishing chaotic systems from stochastic processes [24], detecting noise-induced temporal correlations in stochastic resonance phenomena [47], quantifying the randomness of chaotic pseudo-random number generators [48], discriminating market dynamics [49], and characterizing the complexity of low-frequency fluctuations in semiconductor lasers with optical feedback [50]. In addition, a very related approach, based on computing the number of forbidden patterns present in the time series, has been recently used to find evidence of deterministic behavior in financial time series [51] and to characterize numerically and experimentally the level of stochasticity in the leader-laggard dynamical regime of two mutually coupled semiconductor lasers [52].

\section{NUMERICAL RESUlTS}

In this paper, we focus on the chaotic dynamics of a semiconductor laser. In particular, we consider a single mode laser with moderate delayed feedback, operating in the coherence collapse regime. The data used in our analysis originate from the numerical integration of the widely used LangKobayashi rate equations [53]. These equations have been shown to be successful in modelling the dynamical behaviors of semiconductor lasers subject to weak to moderate coherent optical feedback, taking into account a single reflection in the external cavity. The equations for the complex slowly varying amplitude of the electric field $E(t)$ and the carrier number inside the cavity $N(t)$ are

$$
\begin{aligned}
& \dot{E}(t)=\frac{1+i \alpha}{2}\left[G(t)-\frac{1}{\tau_{p}}\right] E(t)+\gamma E\left(t-\tau_{S}\right) e^{-i \Phi}(5) \\
& \dot{N}(t)=\frac{I}{e}-\frac{N(t)}{\tau_{N}}-G(t)|E(t)|^{2},
\end{aligned}
$$

where $G(t)=g\left(N(t)-N_{0}\right) /\left(1+s|E(t)|^{2}\right)$ is the optical gain. Table I details the different parameters as well as their values as they were used in the simulation. The relaxation oscillation frequency of the solitary laser is $f_{R O}=4.2 \mathrm{GHz}$ at this pumping condition.

\begin{tabular}{|c|c|c|}
\hline Parameter & Description & Value \\
\hline$\alpha$ & linewidth enhancement factor & 5 \\
$\tau_{p}$ & photon lifetime & $2 \mathrm{ps}$ \\
$\tau_{N}$ & carrier lifetime & $2 \mathrm{~ns}$ \\
$g$ & differential gain coefficient & $1.5 \times 10^{-8} \mathrm{ps}^{-1}$ \\
$N_{o}$ & carrier number at transparency & $1.5 \times 10^{8}$ \\
$s$ & gain compression coefficient & $5 \times 10^{-7}$ \\
$\tau_{S}$ & feedback time delay & $1 \mathrm{~ns}$ \\
$\gamma$ & feedback strength & $20 \mathrm{~ns}^{-1}$ \\
$\Phi$ & optical feedback phase & 0 \\
$I_{t h}$ & threshold current & $14.7 \mathrm{~mA}$ \\
$I$ & bias current & $1.5 I_{t h}$ \\
\hline
\end{tabular}

TABLE I

PARAMETER SET IN THE NUMERICAL SIMULATION.

The intensity dynamics of the laser was obtained by numerically integrating Eqs. (7) and (8) using a second-order RungeKutta method with a time step of $\Delta t=0.1$ ps. We analyzed time series of $N=2 \cdot 10^{6}$ data points with a sampling period of $\Omega_{s}=1$ ps. Figure 1 shows a typical temporal trace.

In Fig. 2 we plot the normalized permutation entropy, $\mathcal{H}_{S}$, and the permutation statistical complexity, $\mathcal{C}_{J S}$, associated with the laser intensity time series as a function of the embedding delay $\tau$ for different embedding dimensions $(4 \leq D \leq 8)$. Independently of the embedding dimension the permutation entropy is minimized and the permutation statistical complexity is maximized when the embedding delay $\tau$ of the symbolic reconstruction is similar to $\tau_{S}$, i.e. for $\tau \approx \tau_{s} / \Omega_{s}=1000$. This particular value, denoted as $\tau_{S}^{*}$ hereafter, is slightly larger than $\tau_{s}$ due to the inertia of the laser system. The inertia or internal response time is an inherent property difficult to determine precisely and affects most of the methods proposed to identify time delay from time series [20]. In particular, we have obtained the same time delay estimation by using the autocorrelation function (ACF) and the delayed 


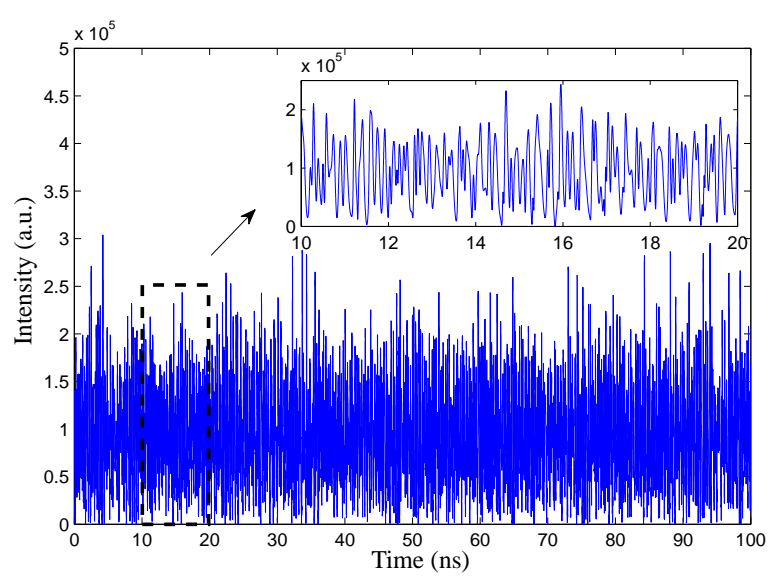

Fig. 1. Numerical chaotic time trace simulated by using the Lang-Kobayashi model at the coherence collapse regime $\left(I=1.5 I_{t h}, \gamma=20 \mathrm{~ns}^{-1}, \tau_{S}=\right.$ $1 \mathrm{~ns}$ and $\Omega_{s}=1 \mathrm{ps}$ ).

mutual information (DMI) since the inertia also affects these conventional techniques [21], [22], [31].

It is worth noting that the time delay of the system can be identified from the analysis of only one of the two quantifiers. Both of them have local extrema around the time delay, providing approximately the same information. However, it should be noted that the permutation statistical complexity is better in identifying the time delay due to the higher contrast with the base line. Other minima and maxima for $\mathcal{H}_{S}$ and $\mathcal{C}_{J S}$, respectively, are obtained when the embedding delay matches harmonics and subharmonics of $\tau_{S}^{*}$. However, they are less pronounced as it can be concluded from Fig. 2. The number of the peaks associated to subharmonics of $\tau_{S}^{*}$ increases with the embedding dimension. More precisely, there are $D-2$ subharmonic peaks for embedding dimension $D$, located at $\tau_{S}^{*} / 2, \tau_{S}^{*} / 3, \ldots, \tau_{S}^{*} /(D-1)$. In the insets of Figure 2 we have detailed the locations of the different peaks for the particular case of embedding dimension $D=8$. It is reasonable to assume that with the largest possible embedding dimension we have considered, i.e. with $D=8$, more information is being included when estimating the quantifiers because, in this case, we are maximizing the length and number of symbols. We just have to take into account that longer time series are necessary in this case $(N \gg D$ !).

From Fig. 2 we can identify other significant extrema of the quantifiers for an embedding delay $\tau$ slightly larger than $\tau_{S}^{*}$ (indicated by the black arrow). The presence of this peak can be attributed to the relaxation oscillation period, $\tau_{R O}$, because its time location $(\tau=1155)$ is approximately equal to $\tau_{S}^{*}+\tau_{R O} / 2$ independently of the embedding dimension. Also for small embedding delays we find the signature of the relaxation oscillation period. The gray arrow indicates the location of a broader peak. Its position is around $\tau_{R O} / 2$. We have confirmed that in the case of periodic functions certain ordinal patterns do not appear, or have very small probabilities, for embedding delay at the half of the period. Consequently, $\mathcal{H}_{\mathcal{S}}$ has a minimum and $\mathcal{C}_{J S}$ has a maximum for this particular embedding delay value. As it can be seen from Fig. 2 the
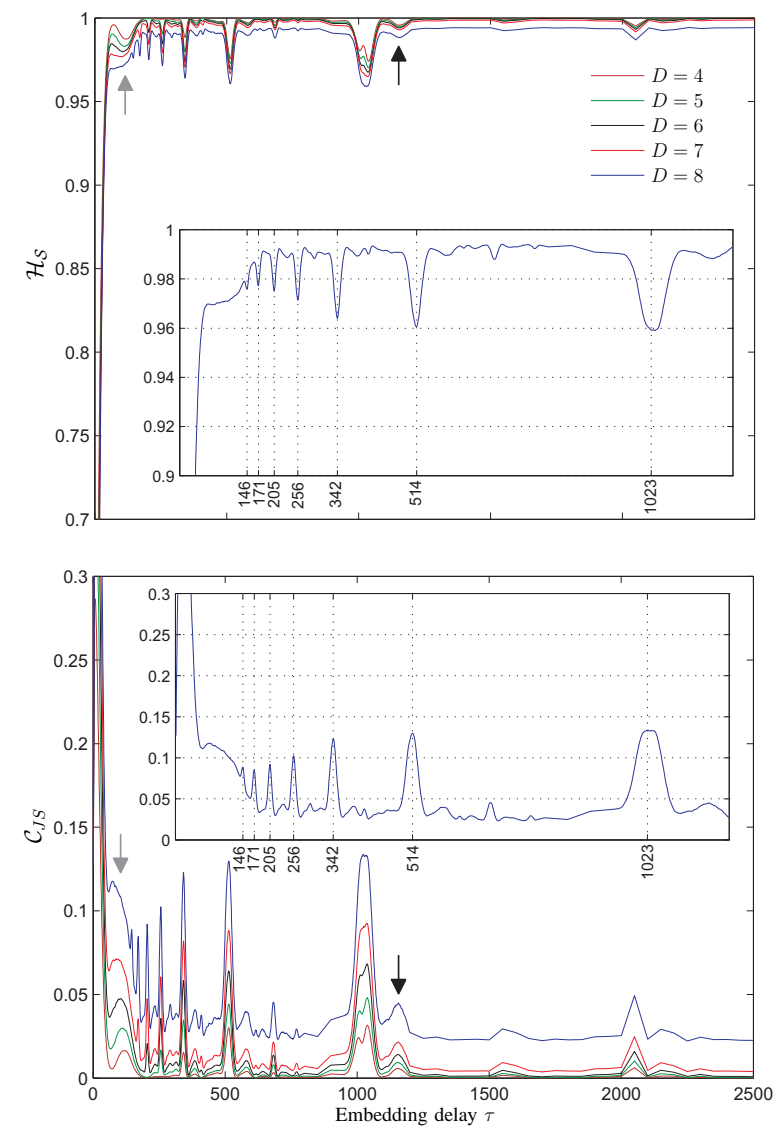

Fig. 2. Permutation entropy $\mathcal{H}_{\mathcal{S}}$ (top) and permutation statistical complexity $\mathcal{C}_{J S}$ (bottom) as a function of the embedding delay $\tau$ with embedding dimensions $4 \leq D \leq 8$ for the numerical intensity time series. Black and gray arrows indicate the peaks associated to the relaxation oscillation period. Locations of the local extrema associated with the feedback time delay $\tau_{S}^{*}$ and its subharmonics for $D=8$ are detailed in the insets. It is worth noting that the local extrema related to subharmonics decrease in amplitude.

location of the latter peak shifts to the left with the embedding dimension and better identification is curiously obtained for smaller embedding dimension values ( $D=4,5$ and 6$)$. We have checked that extrema at similar locations, namely $\tau_{R O} / 2$ and $\tau_{S}^{*}+\tau_{R O} / 2$, are obtained for the autocorrelation function and the delayed mutual information.

In addition, we find a third relevant time scale for an even smaller embedding delay value. The permutation complexity indicator has a pronounced change for well-defined small embedding delays. Figure 3 displays the behavior of both quantifiers for embedding dimensions $4 \leq D \leq 8$ and $1 \leq \tau \leq$ $50 . \mathcal{C}_{J S}$ is maximized for an intermediate value of $\tau$ while $\mathcal{H}_{\mathcal{S}}$ monotonically increases with $\tau$ in this domain highlighting an important difference between both quantifiers. This particular embedding delay value $\tau_{M}$, at which the permutation statistical complexity reaches a local maximum, represents the minimally required sampling rate to capture all the information related to the nonlinear correlations of the fast chaotic dynamics. We note that this time scale is faster than the relaxation oscillation time scale. It is therefore not sufficient to record with the bandwidth of the relaxation oscillations in order to 
acquire the full complexity of the dynamics. The origin of this faster time scale can be associated with the picosecond pulsing due to partial mode locking of the external cavity modes in the delayed feedback system, as it has been found in Ref. [54]. In order to justify that this time scale is related to the fast chaotic dynamics we have analyzed the evolution of the quantifiers for small embedding delays $(1 \leq \tau \leq 50)$ in the complexity-entropy causality plane, i.e. the plane obtained with the permutation entropy of the system in the horizontal axis and the permutation statistical complexity in the vertical one. The term causality takes into consideration that the temporal correlation between successive samples is taken into account by using the permutation probability distribution to estimate both information theory quantifiers. This representation space was shown to be useful to discriminate between chaotic systems and stochastic processes, locating them at different planar positions [24]. It is clear that the embedding delay is directly related to the sampling frequency; i.e. low embedding delay values require high sampling frequencies. For embedding delays smaller than $\tau_{M}, \tau<\tau_{M}$ (sampling frequencies larger than the optimum value) we oversample the dynamics. Thus, spurious and superfluous correlations are introduced, causing low permutation entropy and statistical complexity values typically associated with a regular process (see Fig. 4). On the other hand, for embedding delays larger than $\tau_{M}, \tau>\tau_{M}$ (sampling frequencies smaller than the optimum value) the intrinsic non linear correlations present in the chaotic system are progressively lost due to undersampling. The resulting sampled system resembles a random process with high permutation entropy and low permutation statistical complexity values (see Fig. 4). The curve described by the permutation quantifiers as a function of the embedding delay allows to estimate the amount of information redundancy, determinism, and stochasticity present in the underlying chaotic nature of the laser system. We have checked that the minimally required sampling rate is related to the sampling rate at which other nonlinear time series analysis measures such as correlation dimension provide meaningful results.

It is worth mentioning that De Micco et al. [43] have recently shown that the permutation statistical complexity can be used to determine the best sampling time of chaotic systems by analyzing the behavior of this quantifier as a function of the sampling frequency. They illustrated the results for the case of two paradigmatic examples: the Rössler and Lorenz chaotic attractors. Our approach is slightly different. The original time series of the delayed feedback laser is efficiently subsampled by changing the embedding delay of the symbolic reconstruction, which appears to be a more adequate approach. From Figs. 3 and 4 it can be concluded that $\tau_{M}$ increases with $D$. In Fig. 5 the minimal required sampling time $\tau_{M}$ is plotted as a function of the embedding dimension $D$ for the numerical data. According to this plot, by increasing the embedding dimension $D$ the minimal required sampling time also increases. Therefore, higher values of $D$ allow the use of larger minimal required sampling times, retaining all the information about the chaotic dynamics of the system under analysis. It is necessary to consider that an appropriate statistical analysis can be only done if the number of points of the time series

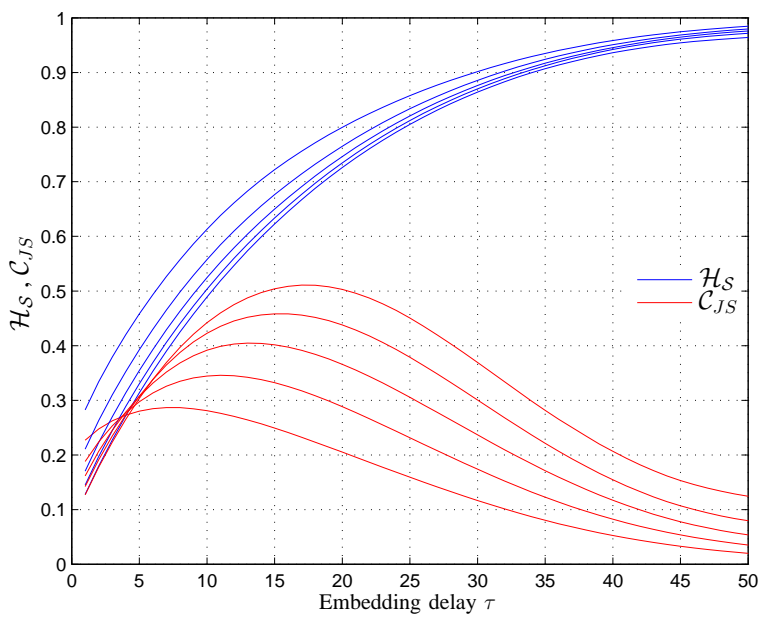

Fig. 3. Permutation entropy $\mathcal{H}_{\mathcal{S}}$ and permutation statistical complexity $\mathcal{C}_{J S}$ as a function of the embedding delay $\tau$ with embedding dimensions $4 \leq$ $D \leq 8$ for the numerical intensity time series. Small embedding time delays are considered $(1 \leq \tau \leq 50)$. $D$ increases from top to bottom for $\mathcal{H}_{\mathcal{S}}$ and from bottom to top for $\overline{\mathcal{C}}_{J S}$.

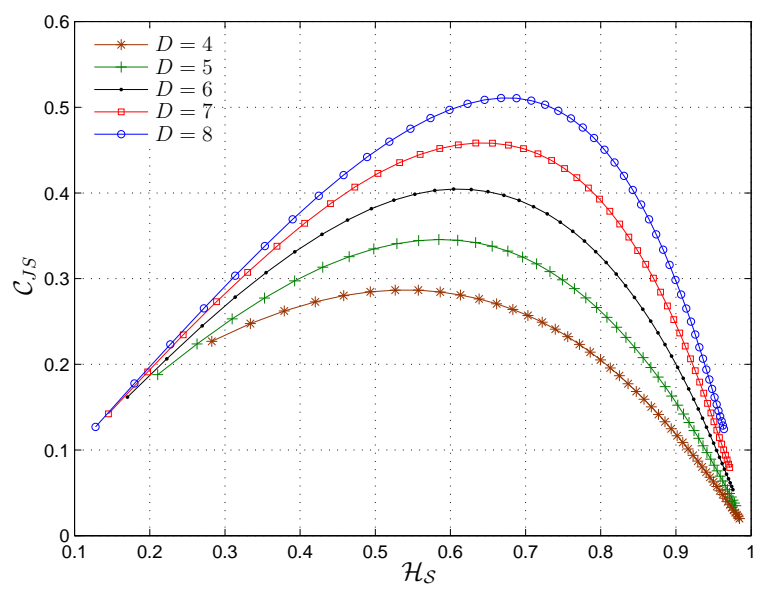

Fig. 4. Evolution of the quantifiers on the complexity-entropy causality plane for the numerical intensity time series as a function of the embedding delay parameter $\tau$ ( $1 \leq \tau \leq 50$, increasing from left to right). Different embedding dimensions $4 \leq D \leq 8$ are considered. A well-defined maximum of $\mathcal{C}_{J S}$ is obtained for an intermediate $\tau$ value.

satisfies $N \gg D$ !. We have found that the values estimated for $\tau_{M}$ are close to the optimal sampling time predicted by the Nyquist-Shannon sampling theorem, even though the chaotic system under study is not a bandwidth-limited signal. As it is depicted in Fig. 6, where the power spectrum of the numerical realization of the dynamical system is plotted, the Nyquist-Shannon theorem predict that the time continuous function is approximately determined and reconstructed with an infinite sequence sampled at $\tau_{N S}=1 /\left(2 f_{\max }\right) \approx 14 \mathrm{ps}$, with $f_{\max }=36 \mathrm{GHz}$. This frequency roughly corresponds to the highest significant frequency in the power spectrum, i.e. $99 \%$ of the full spectrum is taken into account. For smaller cut off frequencies the estimated values for the optimal sampling 


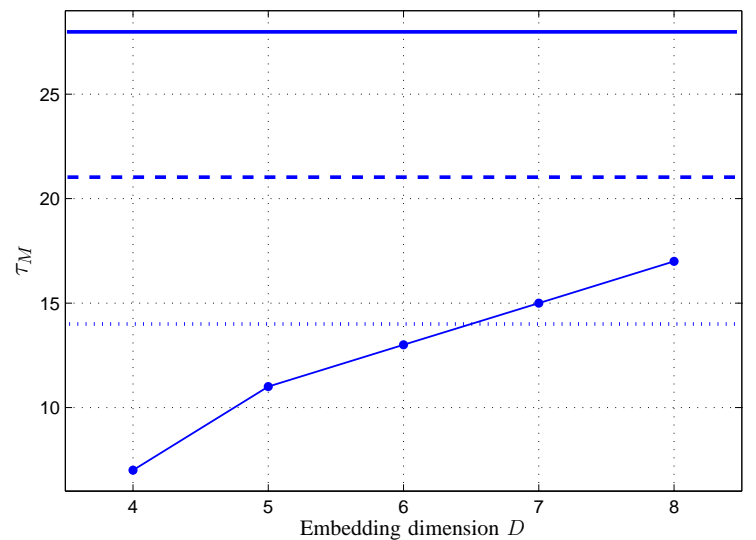

Fig. 5. Minimal required sampling time $\tau_{M}$ as a function of the embedding dimension $D$. The horizontal lines corresponds to the optimal sampling time predicted by the Nyquist-Shannon sampling theorem taking into account the 90\% (solid line, $28 \mathrm{ps}$ ), 95\% (dashed line, $21 \mathrm{ps)}$ ) and 99\% (dotted line, $14 \mathrm{ps}$ ) of the full spectrum.

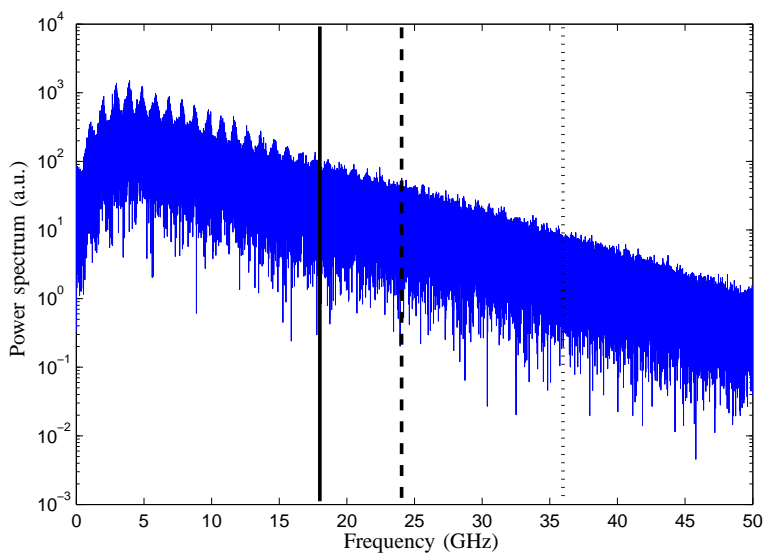

Fig. 6. Power spectrum of the analyzed numerical intensity time series. Vertical lines indicate the location of the different cut off frequencies at the $90 \%$ (solid line, $18 \mathrm{GHz}$ ), $95 \%$ (dashed line, $24 \mathrm{GHz}$ ) and $99 \%$ (dotted line, $36 \mathrm{GHz})$ of the full spectrum.

time increase. They are around 21 ps and 28 ps when the $95 \%$ and $90 \%$ of the full power spectrum, respectively, is considered (see Figs. 5 and 6).

\section{EXPERIMENTAL RESULTS}

Experiments on the delayed feedback dynamics of a semiconductor laser were performed using a fiber pigtailed semiconductor laser lasing at $1542 \mathrm{~nm}$, fabricated by Eblana Photonics. The threshold current of the solitary laser is $I_{t h}=$ $11.7 \mathrm{~mA}$ at $20{ }^{\circ} \mathrm{C}$. The laser exhibits single-mode emission above the lasing threshold. The side-mode suppression ratio of this device is over $40 \mathrm{~dB}$ when the laser is biased at $I=18 \mathrm{~mA}$. The temperature is stabilized up to $\pm 0.01 \mathrm{~K}$. This device has been packaged without an optical isolator so that optical feedback studies can be performed.

The external optical feedback has been introduced using a fiber loop, such that the laser operates in the long cavity

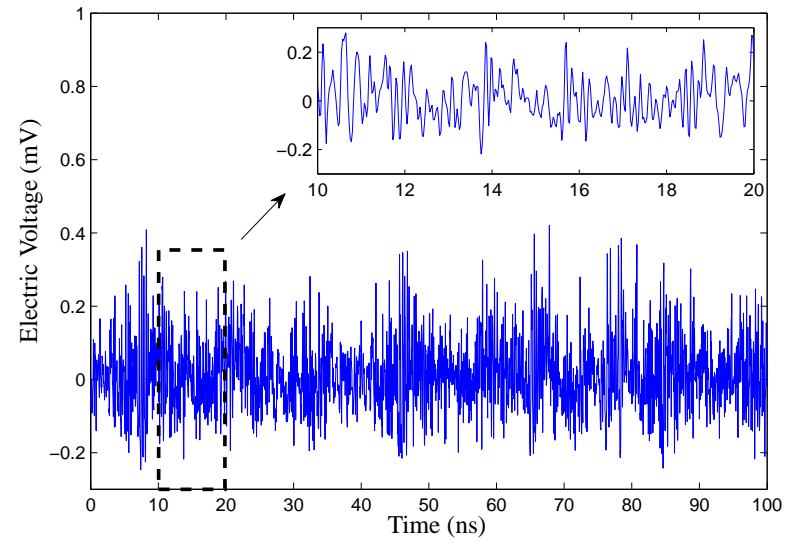

Fig. 7. Experimental chaotic time trace recorded by using a $16 \mathrm{GHz}$ bandwidth digital scope with a sampling rate of 40 Gsamples/second $\left(\delta_{s}=25 \mathrm{ps}\right)$.

regime [55]. This regime is defined by the time delays of the feedback loop being much longer than the relaxation oscillation period. In our experiment, the length of the external fiber cavity is about $L_{e x t}=3.5 \mathrm{~m}$, i.e. the round trip time delay is estimated to be around $\tau_{e x t}=\frac{2 n L_{e x t}}{c}=38.5 \mathrm{~ns}$, where $n$ is the refractive index in the optical fiber and $c$ is the speed of light. When the laser is biased at $I=18 \mathrm{~mA}$, the relaxation oscillation period is $T_{R O}=0.24 \mathrm{~ns}$, which is much shorter than the time delay. The threshold current of the laser is reduced to $10.33 \mathrm{~mA}$ (12\% threshold reduction) when the feedback fiber loop is optimized.

The intensity dynamics is detected via an AC-coupled $13 \mathrm{GHz}$ bandwidth photodiode (Miteq DR-125G-A). The converted electrical signal is then analyzed using a $16 \mathrm{GHZ}$ bandwidth digital scope with a sampling rate of $40 \mathrm{GSamples} / \mathrm{s}$ (LeCroy WaveMaster 816Zi) and by a spectrum analyzer with a $9 \mathrm{kHz}-30 \mathrm{GHz}$ bandwidth (Anritsu MS2667C). This is close to the current technology limit for temporal detection of long time series, with a sampling time of $\delta_{s}=25 \mathrm{ps}$. Time series with $N=2 \cdot 10^{6}$ data points were recorded. We note that different sampling rates are selected in the numerical and experimental analysis. This is because the small sampling period we have chosen in the numerical study can not be experimentally attained.

The detected time trace of the intensity dynamics for a bias current of $I=18 \mathrm{~mA}$ is shown in Fig. 7. The dynamical time scales of the laser in the coherence collapse regime [56] are associated with the relaxation oscillation frequency of several GHz. Therefore, we can sufficiently resolve the temporal dynamics of the laser output with the sampling time and frequency resolution of our detection scheme. The fast intensity dynamic of the laser displays irregular oscillations [54], as it can be seen in the inset of Fig. 7. The temporal separation among individual pulses is in a range of 200 to 400 ps.

In Fig. 8 we plot the normalized permutation entropy and the permutation statistical complexity obtained from the experimental time series as a function of the embedding delay $\tau$ for an embedding dimension $D=8$. We verify experimentally that the permutation entropy is minimized and 
the permutation statistical complexity maximized when the embedding delay $\tau$ of the symbolic reconstruction takes values near $\tau_{\text {ext }}$, i.e. for $\tau$ close to $1540\left(\tau_{\text {ext }} / \delta_{s}=1540\right)$. We have also found the other extrema when the embedding delay matches harmonics and subharmonics of $\tau_{\text {ext }}$. In analogy to the numerical case, they are less noticeable. The differences in peak resolution found when comparing Fig. 2 and Fig. 8 are due to the different sampling periods. We consider that these experimental results confirm the reliability and robustness of our permutation information theory approach to identify the feedback time delay in a real situation.

We have also analyzed the permutation information quantifiers for small embedding delays looking for the other relevant fast time scales of the laser. As it can be seen from Fig. 9, for the current experimental sampling time $\left(\delta_{s}=25 \mathrm{ps}\right)$ the permutation information quantifiers take values near the optimal ones, i.e. $\mathcal{H}_{\mathcal{S}} \approx 0.7$ and $\mathcal{C}_{J S} \approx 0.5$, for the smallest embedding delay $(\tau=1)$ and the largest embedding dimension $(D=8)$. Comparing Figs. 3 and 9, we conclude that the experimental sampling time is very close to the minimal required sampling time for an embedding dimension $D=8$. Numerical and experimental results are not directly comparable because $\tau_{\text {ext }} \gg \tau_{S}$. However, we have numerically checked that the minimal required sampling time is the same for different feedback delays $\tau_{s}$ in the long cavity regime ( $250 \mathrm{ps}, 500 \mathrm{ps}, 1 \mathrm{~ns}, 10 \mathrm{~ns}, 20 \mathrm{~ns}, 30 \mathrm{~ns}$, and $40 \mathrm{~ns}$ ). For these different feedback time delays there is hardly any change in the chaotic bandwidth. Consequently, we find that the minimal required sampling time, directly related to the fastest relevant time scales in the system, is independent of the feedback delay time in this regime. The signature of the relaxation oscillation period appears around $\tau=4$, as it is shown in the inset of Fig. 9. Notice the vertical enlargement necessary to unveil the presence of the extremum.

In order to demonstrate experimentally the presence of the maximum of the permutation statistical complexity for small embedding delays we have analyzed experimental chaotic time traces obtained with lower bias current $(I=13 \mathrm{~mA})$ and feedback strength, where the bandwidth of the chaotic system decreases. Hence, the minimal required sampling time should increase. As it can be seen in Fig. 10, a clear maximum for $\mathcal{C}_{J S}$ is found for a small embedding delay $\left(\tau_{M}=2\right)$ while $\mathcal{H}_{\mathcal{S}}$ is an increasing function of $\tau$ in this range. This is an experimental confirmation of the identification of the fast time scale of the laser with the permutation information analysis. The other extrema observed in Fig. 10 for both quantifiers when $\tau=8$ are associated to the relaxation oscillation period $\left(T_{R O}\right)$. For this lower bias current we have found that $f_{R O} \approx 2.2 \mathrm{GHz}$. Then, the location of the extrema is nearly $T_{R O} / 2$ supporting the relaxation oscillation signature found in the numerical analysis.

\section{CONCLUSIONS}

We have shown both numerically and experimentally that a permutation information theory analysis, based on the estimations of permutation entropy and statistical complexity, is able to identify characteristic time scales present in the chaotic
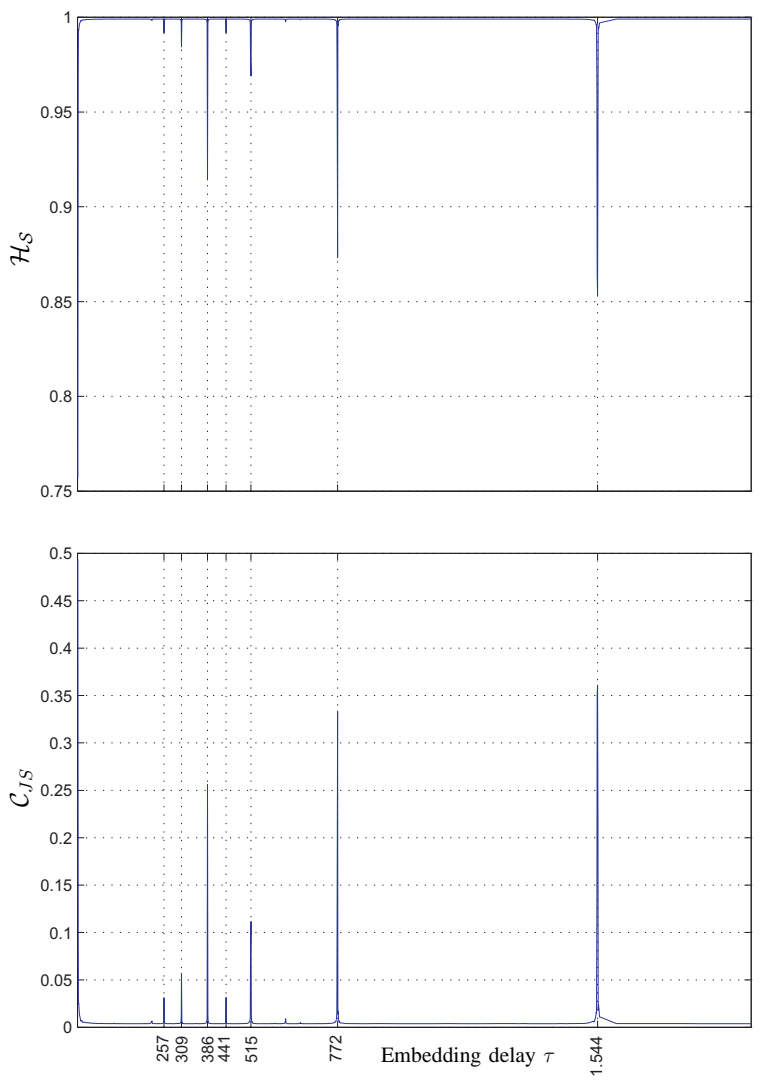

Fig. 8. Permutation entropy $\mathcal{H}_{\mathcal{S}}$ (top) and permutation statistical complexity $\mathcal{C}_{J S}$ (bottom) as a function of the embedding delay $\tau$ with embedding dimensions $D=8$ for the experimental time series. Location of the local extrema associated with the feedback time delay $\tau_{S}^{*}$ and its subharmonics are detailed. Notice that the extremum obtained for $\tau=441$ corresponds to a subharmonic of $2 \tau_{S}^{*}$.

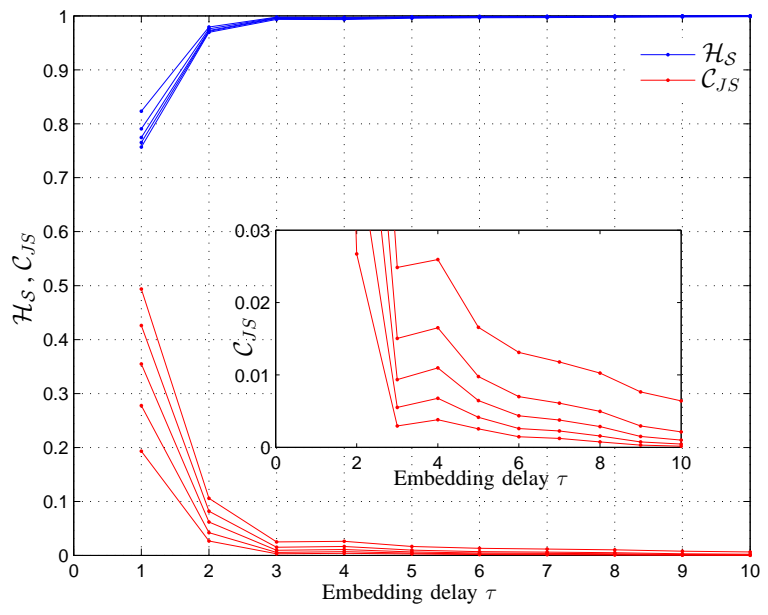

Fig. 9. Permutation entropy $\mathcal{H}_{\mathcal{S}}$ and permutation statistical complexity $\mathcal{C}_{J S}$ as a function of the embedding delay $\tau$ with embedding dimensions $4 \leq D \leq$ 8 for the experimental intensity time series. Small embedding time delays are considered $(1 \leq \tau \leq 10)$. $D$ increases from top to bottom for $\mathcal{H}_{\mathcal{S}}$ and from bottom to top for $\overline{\mathcal{C}}_{J S}$. The relaxation oscillation signature is shown in the inset. 


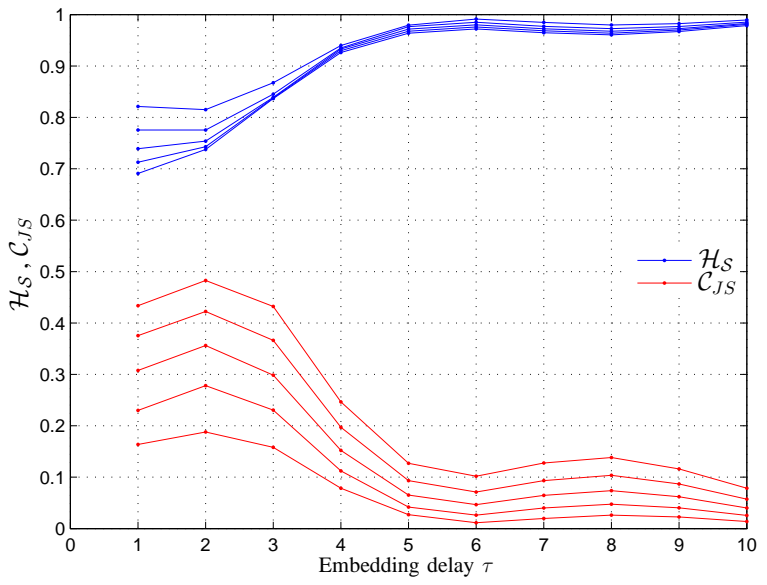

Fig. 10. Permutation entropy $\mathcal{H}_{\mathcal{S}}$ and permutation statistical complexity $\mathcal{C}_{J S}$ as a function of the embedding delay $\tau$ with embedding dimensions $4 \leq$ $D \leq 8$ for the experimental intensity time series with lower bias current $(I=$ $13 \mathrm{~mA}$ ) and feedback strength. Small embedding time delays are considered $(1 \leq \tau \leq 10)$. $D$ increases from top to bottom for $\mathcal{H}_{\mathcal{S}}$ and from bottom to top for $\overline{\mathcal{C}}_{J S}$.

dynamics of a semiconductor laser subject to optical feedback. By analyzing the behavior of these quantifiers as a function of the embedding delay of the symbolic reconstruction it is possible to identify the feedback time delay, the relaxation oscillation period and the picosecond pulsing time scale of this relevant physical system. On the one hand, the feedback time delay and the relaxation oscillation period are associated with embedding delay values that minimize the permutation entropy and maximize the permutation statistical complexity, simultaneously. The presence of additional peaks at harmonics and subharmonics of the feedback time delay allow us to distinguish between these two intrinsic time scales. On the other hand, the fastest time scale defining the minimal required sampling time can be estimated as the embedding delay value where the permutation statistical complexity is also maximized while the permutation entropy has a monotone increasing behavior around this domain. According to these results estimations of both quantifiers are necessary to identify all the relevant time scales. Moreover, we have also found that the minimal required sampling rate decreases when the embedding dimension is increased. Thus, all the information of the chaotic system is retained with a smaller sampling frequency by increasing the embedding dimension. This finding can be very valuable for experimental analysis. Our analysis confirms that high bandwidth and high sampling rates beyond the relaxation oscillation bandwidth are required to allow for a full time series analysis of the chaotic semiconductor laser dynamics. Fortunately, these high experimental demands have finally come into reach and promise further interesting insights into the complex dynamics of semiconductor lasers and its functional utilization.

\section{ACKNOWLEDGMENT}

M.C.S. thanks the MEC for a "Juan de la Cierva" contract. L.Z. and O.A.R. were supported by Consejo Nacional de
Investigaciones Científicas y Técnicas (CONICET), Argentina. O.A.R. is PVE fellowship, CAPES, Brazil. Part of this work was funded by MEC (Spain), MICINN (Spain) and FEDER under Projects TEC2006-10009/MIC (PhoDeCC), TEC200914101 (DeCoDicA) and FIS2007-60327 (FISICOS), and by the EC Project PHOCUS Grant 240763.

\section{REFERENCES}

[1] I. Fischer, O. Hess, W. Elsässer, and E. Göbel, "High-dimensional chaotic dynamics of an external cavity semiconductor laser," Phys. Rev. Lett., vol. 73, pp. 2188-2191, 1994.

[2] R. Vicente, J. Daudén, P. Colet, and R. Toral, "Analysis and characterization of the hyperchaos generated by a semiconductor laser subject to a delayed feedback loop," IEEE J. Quantum Electron., vol. 41, pp. 541-548, 2005.

[3] A. Pikovsky, M. Rosenblum, and J. Kurths, Synchronization: A Universal Concept in Nonlinear Science. Cambridge University Press, 2001.

[4] F.-Y. Lin and J.-M. Liu, "Diverse waveform generation using semiconductor lasers for radar and microwave applications," IEEE J. Quantum Electron., vol. 40, pp. 682-689, 2004.

[5] — "Chaotic lidar," IEEE J. Sel. Topics Quantum Electron., vol. 10, pp. 991-997, 2004.

[6] M. Peil, I. Fischer, W. Elsässer, S. Bakić, N. Damaschke, C. Tropea, S. Stry, and J. Sacher, "Rainbow refractometry with a tailored incoherent semiconductor laser source," Appl. Phys. Lett., vol. 89, p. 091106, 2006.

[7] A. Uchida, K. Amano, M. Inoue, K. Hirano, S. Naito, H. Someya, I. Oowada, T. Kurashige, M. Shiki, S. Yoshimori, K. Yoshimura, and P. Davis, "Fast physical random bit generation with chaotic semiconductor lasers," Nat. Photon., vol. 2, pp. 728-732, 2008.

[8] T. E. Murphy and R. Roy, "Chaotic lasers: the world's fastest dice," Nat. Photon., vol. 2, pp. 714-715, 2008.

[9] C. R. Mirasso, P. Colet, and P. Garcia-Fernandez, "Synchronization of chaotic semiconductor lasers: application to encoded communications," IEEE Photon. Technol. Lett., vol. 8, pp. 299-301, 1996.

[10] V. Annovazzi-Lodi, S. Donati, and A. Sciré, "Synchronization of chaotic injected-laser systems and its application to optical cryptography," IEEE J. Quantum Electron., vol. 32, pp. 953-959, 1996.

[11] G. D. VanWiggeren and R. Roy, "Communication with chaotic lasers," Science, vol. 279, pp. 1198-1200, 1998.

[12] J. Ohtsubo, "Chaos synchronization and chaotic signal masking in semiconductor lasers with optical feedback," IEEE J. Quantum Electron., vol. 38, pp. 1141-1154, 2002.

[13] A. Argyris, D. Syvridis, L. Larger, V. Annovazzi-Lodi, P. Colet, I. Fischer, J. Garca-Ojalvo, C. R. Mirasso, L. Pesquera, and K. A. Shore, "Chaos-based communications at high bit rates using commercial fibreoptic links," Nature, vol. 438, pp. 343-346, 2005.

[14] A. Wang, Y. Wang, and H. He, "Enhancing the bandwidth of the optical chaotic signal generated by a semiconductor laser with optical feedback," IEEE Photon. Technol. Lett., vol. 20, pp. 1633-1635, 2008.

[15] R. Hegger, M. J. Bünner, H. Kantz, and A. Giaquinta, "Identifying and modeling delay feedback systems," Phys. Rev. Lett., vol. 81, pp. 558561, 1998.

[16] H. Voss and J. Kurths, "Reconstruction of nonlinear time-delayed feedback models from optical data," Chaos, Solitons \& Fractals, vol. 10 pp. 805-809, 1999.

[17] C. Zhou and C.-H. Lai, "Extracting messages masked by chaotic signals of time-delay systems," Phys. Rev. E, vol. 60, pp. 320-323, 1999.

[18] V. S. Udaltsov, L. Larger, J. P. Goedgebuer, A. Locquet, and D. S. Citrin, "Time delay identification in chaotic cryptosystems ruled by delay-differential equations," J. Opt. Technol., vol. 72, pp. 373-377, 2005.

[19] M. C. Soriano, P. Colet, and C. R. Mirasso, "Security implications of open- and closed-loop receivers in all-optical chaos-based communications," IEEE Photon. Technol. Lett., vol. 21, pp. 426-428, 2009.

[20] S. Ortín González, "Reconstrucción de la dinámica no lineal de sistemas caóticos con retraso mediante redes neuronales," Ph.D. dissertation, Universidad de Cantabria, España, 2009, in Spanish.

[21] D. Rontani, A. Locquet, M. Sciamanna, and D. S. Citrin, "Loss of time-delay signature in the chaotic output of a semiconductor laser with optical feedback," Opt. Lett., vol. 32, pp. 2960-2962, 2007.

[22] D. Rontani, A. Locquet, M. Sciamanna, D. S. Citrin, and S. Ortin, "Time-delay identification in a chaotic semiconductor laser with optical feedback: a dynamical point of view," IEEE J. Quantum Electron., vol. 45, pp. 879-891, 2009. 
[23] L. Zunino, M. C. Soriano, I. Fischer, O. A. Rosso, and C. R. Mirasso, "Permutation information theory approach to unveil delay dynamics from time series analysis," Submitted to Phys. Rev. E, 2010.

[24] O. A. Rosso, H. A. Larrondo, M. T. Martín, A. Plastino, and M. A. Fuentes, "Distinguishing noise from chaos," Phys. Rev. Lett., vol. 99, p. 154102, 2007.

[25] J. M. Amigó, S. Zambrano, and M. A. F. Sanjuán, "True and false forbidden patterns in deterministic and random dynamics," Europhys. Lett., vol. 79, p. 50001, 2007.

[26] J.-G. Wu, G.-Q. Xia, L.-P. Cao, and Z.-M. Wu, "Experimental investigations on the external cavity time signature in chaotic output of an incoherent optical feedback external cavity semiconductor laser," Opt. Commun., vol. 282, pp. 3153-3156, 2009.

[27] J.-G. Wu, G.-Q. Xia, and Z.-M. Wu, "Suppression of time delay signatures of chaotic output in a semiconductor laser with double optical feedback," Opt. Express, vol. 17, pp. 20 124-20 133, 2009.

[28] A. C. Fowler and G. Kember, "Delay recognition in chaotic time series," Phys. Lett. A, vol. 175, pp. 402-408, 1993.

[29] Y.-C. Tian and F. Gao, "Extraction of delay information from chaotic time series based on information entropy," Physica D, vol. 108, pp. 113-118, 1997.

[30] M. Lehrman, A. B. Rechester, and R. B. White, "Symbolic analysis of chaotic signals and turbulent fluctuations," Phys. Rev. Lett., vol. 78, pp. 54-57, 1997.

[31] M. J. Bünner, A. Kittel, J. Parisi, I. Fischer, and W. Elsässer, "Estimation of delay times from a delayed optical feedback laser experiment," Europhys. Lett., vol. 42, pp. 353-358, 1998.

[32] R. K. Azad, J. S. Rao, and R. Ramaswamy, "Information-entropic analysis of chaotic time series: determination of time-delays and dynamical coupling," Chaos, Solitons \& Fractals, vol. 14, pp. 633-641, 2002.

[33] S. Ortín, J. M. Gutiérrez, L. Pesquera, and H. Vasquez, "Nonlinear dynamics extraction for time-delay systems using modular neural networks synchronization and prediction," Physica A, vol. 351, pp. 133-141, 2005.

[34] M. Siefert, "Practical criterion for delay estimation using random perturbations," Phys. Rev. E, vol. 76, p. 026215, 2007.

[35] M. D. Prokhorov and V. I. Ponomarenko, "Reconstruction of time-delay systems using small impulsive disturbances," Phys. Rev. E, vol. 80, p. 066206, 2009

[36] C. Bandt and B. Pompe, "Permutation entropy: a natural complexity measure for time series," Phys. Rev. Lett., vol. 88, p. 174102, 2002.

[37] D. P. Feldman, C. S. McTague, and J. P. Crutchfield, "The organization of intrinsic computation: complexity-entropy diagrams and the diversity of natural information processing," Chaos, vol. 18, p. 043106, 2008.

[38] P. W. Lamberti, M. T. Martín, A. Plastino, and O. A. Rosso, "Intensive entropic non-triviality measure," Physica A, vol. 334, pp. 119-131, 2004

[39] R. López-Ruiz, H. L. Mancini, and X. Calbet, "A statistical measure of complexity," Phys. Lett. A, vol. 209, pp. 321-326, 1995

[40] I. Grosse, P. Bernaola-Galván, P. Carpena, R. Román-Roldán, J. Oliver, and H. E. Stanley, "Analysis of symbolic sequences using the JensenShannon divergence," Phys. Rev. E, vol. 65, p. 041905, 2002.

[41] M. T. Martín, A. Plastino, and O. A. Rosso, "Generalized statistical complexity measures: geometrical and analytical properties," Physica $A$, vol. 369, pp. 439-462, 2006.

[42] J. R. Sánchez and R. López-Ruiz, "A method to discern complexity in two-dimensional patterns generated by coupled map lattices," Physica $A$, vol. 355, pp. 633-640, 2005.

[43] L. D. Micco, J. G. Fernández, H. A. Larrondo, A. Plastino, and O. A. Rosso, "Statistical complexity of sampled chaotic attractors," Submitted to Physica D, 2010.

[44] M. Staniek and K. Lehnertz, "Parameter selection for permutation entropy measurements," Int. J. of Bifurcation and Chaos, vol. 17, pp. 3729-3733, 2007.

[45] Y. Cao, W. Tung, J. B. Gao, V. A. Protopopescu, and L. M. Hively, "Detecting dynamical changes in time series using the permutation entropy," Phys. Rev. E, vol. 70, p. 046217, 2004.

[46] L. Zunino, D. G. Pérez, M. T. Martín, M. Garavaglia, A. Plastino, and O. A. Rosso, "Permutation entropy of fractional Brownian motion and fractional Gaussian noise," Phys. Lett. A, vol. 372, pp. 4768-4774, 2008

[47] O. A. Rosso and C. Masoller, "Detecting and quantifying stochastic and coherence resonances via information-theory complexity measurements," Phys. Rev. E, vol. 79, p. 040106(R), 2009.

[48] L. D. Micco, H. A. Larrondo, A. Plastino, and O. A. Rosso, "Quantifiers for randomness of chaotic pseudo-random number generators," Phil. Trans. R. Soc. A, vol. 367, pp. 3281-3296, 2009.

[49] L. Zunino, M. Zanin, B. M. Tabak, D. G. Pérez, and O. A. Rosso, "Complexity-entropy causality plane: a useful approach to quantify the stock market inefficiency," Physica A, vol. 389, pp. 1891-1901, 2010.
[50] J. Tiana-Alsina, M. C. Torrent, O. A. Rosso, C. Masoller, and J. GarcíaOjalvo, "Quantifying the statistical complexity of low-frequency fluctuations in semiconductor lasers with optical feedback," Phys. Rev. A, vol. 82, p. 013819,2010 .

[51] M. Zanin, "Forbidden patterns in financial time series," Chaos, vol. 18, p. 013119, 2008.

[52] J. Tiana-Alsina, J. M. Buldú, M. C. Torrent, and J. García-Ojalvo, "Quantifying stochasticity in the dynamics of delay-coupled semiconductor lasers via forbidden patterns," Phil. Trans. R. Soc. A, vol. 368, pp. 367-377, 2010.

[53] R. Lang and K. Kobayashi, "External optical feedback effects on semiconductor injection laser properties," IEEE J. Quantum Electron., vol. 16 , pp. $347-355,1980$.

[54] I. Fischer, G. H. M. van Tartwijk, A. M. Levine, W. Elsässer, E. Göbel, and D. Lenstra, "Fast pulsing and chaotic itinerancy with a drift in the coherence collapse of semiconductor lasers," Phys. Rev. Lett., vol. 76, pp. 220-223, 1996.

[55] T. Heil, I. Fischer, W. Elsässer, and A. Gavrielides, "Dynamics of semiconductor lasers subject to delayed optical feedback: the short cavity regime," Phys. Rev. Lett., vol. 87, p. 243901, 2001.

[56] D. Lenstra, B. Verbeek, and A. D. Boef, "Coherence collapse in singlemode semiconductor lasers due to optical feedback," IEEE J. Quantum Electron., vol. 21, pp. 674-679, 1985.

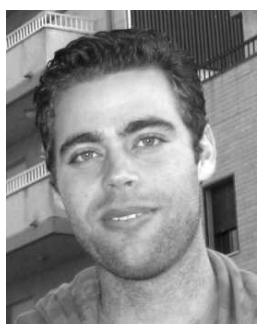

Miguel Cornelles Soriano was born in Benicarlo, Spain, in 1979. He received the Telecom Engineering degree from the Technical University of Catalonia, Spain, in 2002 and the Ph.D. degree in Engineering from the Free University of Brussels, Belgium, in 2006. Since January 2008, he holds a "Juan de la Cierva" scientific contract at the Instituto de Física Interdisciplinar y Sistemas Complejos (IFISC). His main research interests include optical feedback, theoretical modeling and synchronization properties of semiconductor lasers.

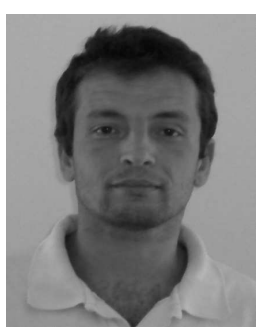

Luciano Zunino was born in Chivilcoy, Buenos Aires, Argentina, in 1975. He received the M.Sc. and Ph.D. degrees in Physics from the Universidad Nacional de La Plata, Buenos Aires, Argentina, in 2000 and 2005, respectively. Since April 2007, he has held a permanent research position at the Argentinean Consejo Nacional de Investigaciones Científicas y Técnicas (CONICET). His main research interests include time series analysis, nonlinear dynamics, econophysics and light propagation in turbulent media.

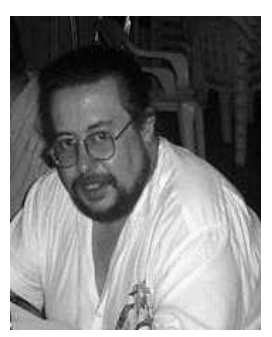

Osvaldo A. Rosso was born in Rojas, Buenos Aires, Argentina, on October 1954. He received the M.Sc. and Ph.D. degrees in Physics from the Universidad Nacional de La Plata, Buenos Aires, Argentina, in 1978 an 1984, respectively. He held a post-doc position at Forschungzentrum Jülich GmBH (KFA), Jülich Germany (1988-1990). Visiting Researcher at Istituto Lamel, Sezione di Cinematografia Scientifica, CNR, Bologna, Italy (1990-1992). Research Academic, School of Electrical Engineering and Computer Science, The University of Newcastle, Newcastle, Australia (2007-2009). At present he is Professor Visitante do Exterior (PVE), categoria Doutor Senior, CAPES, Brazil (2010). Since March 1985, he has held a permanent research position at the Argentinean Consejo Nacional de Investigaciones Científicas y Técnicas (CONICET). He has authored or coauthored over 130 publications including about 85 journal papers. His main research interests include time series analysis, nonlinear dynamics, Information Theory, Time-Frequency Analysis and, their applications to physics, biological and medical sciences. 


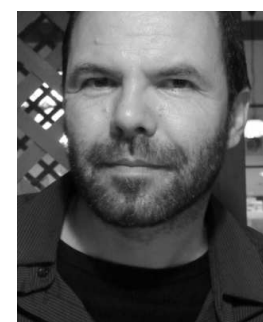

Ingo Fischer received his diploma and Ph.D. degrees in physics from Philipps University, Marburg, Germany, in 1992 and 1995, respectively. He stayed at TU Darmstadt, Germany, from 1995 to 2004 and at Vrije Universiteit Brussel, Belgium, from 2005 to 2007. In 2007 he became full professor in Photonics at Heriot-Watt University, Edinburgh, U.K. Since 2009 he is professor at the Institute for CrossDisciplinary Physics and Complex Systems, IFISC (UIB-CSIC). His studies are focused on nonlinear photonics and brain dynamics. In particular, he is concentrating on emission properties and dynamics of modern photonic sources, coupled laser systems, synchronization of lasers and neurons, and utilization of chaos. He received the Research Prize of the Adolf-Messer Foundation in 2000 and the first Hassian Cooperation Prize of the Technology Transfer Network in 2004.

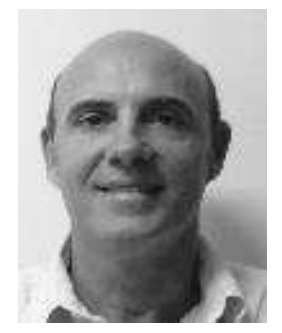

Claudio R. Mirasso was born in Buenos Aires, Argentina, in 1960. He received the M.Sc. and Ph.D. degrees in Physics from the Universidad Nacional de La Plata, Buenos Aires, Argentina, in 1984 and 1989, respectively. After several Postdoctoral positions in Spain and the Netherlands, he became an Associate Professor with the Physics Department, Universiteit de les Illes Balears (UIB), in 1996. He has authored or coauthored over 140 publications including about 110 journal papers. His research interests include instabilities in semiconductor lasers, synchronization, and control of chaotic semiconductor lasers, dynamics and applications of delayed coupled semiconductor lasers, and applications of nonlinear dynamics. 